Depuis plusieurs années,

les démarches dites

" d'investigation » sont mises

en avant dans les programmes

scolaires en mathématiques

et en sciences expérimentales.

Depuis septembre 2013,

les lycéens arrivant dans

l'enseignement supérieur

ont été initiés à ces démarches,

en particulier à l'école primaire

et au collège [1].

Lors du minicolloque

" Enseignement » du congrès

général de la SFP 2013, nous

nous sommes centrés sur ce

nouveau " profil » d'étudiant

qui nécessite d'être connu

des enseignants du supérieur,

en particulier concernant

les démarches scientifiques.

\title{
Démarches d'investigation en sciences et construction du savoir
}

\author{
Cécile de Hosson $^{(1,8)}$, Estelle Blanquet ${ }^{(2,3,8)}$, Éric Picholle ${ }^{(4)}$, Valérie Munier ${ }^{(5)}$, \\ Alice Delserieys $^{(6)}$ et Nathalie Lebrun ${ }^{(1,7,8)}$ (nathalie.lebrun@univ-lille1.fr) \\ (1) LDAR (EA 147), Université Paris Diderot \\ (2) ESPE de Nice, GIS « CAPEF », Université de Nice Sophia-Antipolis \\ (3) IUFE, LDES, Université de Genève \\ (4) LMPC (UMR7336), CNRS, Université de Nice Sophia Antipolis \\ (5) LIRDEF, Universités Montpellier 2/3, ESPE Montpellier \\ (6) ADEF EA 4671, Aix-Marseille Université \\ (7) Université Lille 1 \\ (8) Commission Enseignement, Société Française de Physique
}

\section{La démarche d'investigation (DI) dans les programmes scolaires: éléments d'une genèse}

L'enseignement fondé sur l'investigation s'est installé en tant qu'approche pédagogique pour l'enseignement des sciences dans la plupart des pays du monde. Les racines de cet engagement massif de l'enseignement des sciences (de l'école primaire jusqu'au lycée) vers l'investigation sont multiples et de nature variée ; les lignes qui suivent, sans prétendre à l'exhaustivité, visent à retracer à grands traits l'histoire de ce qu'il est convenu d'appeler en France la " démarche d'investigation " (DI, voir encadré).

Avant les années 2000, l'enseignement des sciences à l'école primaire semble avoir déserté les classes. Cet argument constitue l'un des leviers du Plan de Rénovation de l'Enseignement des Sciences et de la Technologie (PRESTE), mis en place en 2000 par l'Inspection générale de l'Éducation nationale, suite à un travail de terrain réalisé par l'Inspection dans les écoles " pilotes » qui travaillaient depuis quelques mois en collaboration avec La main à la pâte ${ }^{(a)}$. C'est dans le PRESTE que le mot "investigation » apparait pour la première fois, pour qualifier l'approche pédagogique qui allait, dès lors, être privilégiée.

Quelques années plus tard, était publié le " socle commun des connaissances et des compétences ", en même temps que les nouveaux programmes de collège. La DI devient, en tant que label, l'approche pédagogique à favoriser pour l'enseignement des sciences (expérimentales et mathématiques). Si l'on rentre un peu dans les détails, on s'aperçoit que le choix des concepteurs des programmes du collège se porte sur la notion de "situation problème ", notion dont la création remonte aux années 70 et à la réforme des cursus canadiens de médecine. La DI situe l'apprentissage des sciences dans une approche bachelardienne de résolution de problème et de franchissement d'obstacle [2]. La prise en compte des " conceptions des élèves ", ces idées pouvant faire obstacle à l'apprentissage scientifique, apparait comme une nécessité, leur connaissance par les enseignants également. Plus récemment, c'est le lycée qui a vu apparaître la DI comme pratique pédagogique d'enseignement des sciences.

L'installation de la DI (ou plus largement, de l'enseignement des sciences fondé sur l'investigation) dans les programmes scolaires a certainement profité d'influences de natures diverses. Au-delà du rôle joué par La main à la pâte, une autre influence peut être recherchée du côté de l'Union Européenne. En 2007, dans le "Rapport Rocard » [3], un lien est établi entre la désaffection des étudiants pour les filières scientifiques et la nécessité de changement de méthode pédagogique pour l'enseignement des sciences. Les bienfaits de la DI sont posés comme des certitudes et conduisent les auteurs à conclure à la nécessité de l'étendre à l'ensemble des écoles du territoire européen. Les résultats des évaluations interna- 
tionales PISA contribuent également à cette émergence. Et, en effet, si l'on examine la nature de ce qu'évaluent les tests PISA, on retrouve des aspects des savoirs de sciences et des aspects de savoirs sur la science qui incluent des démarches scientifiques auxquelles sont associées les DI. Bien entendu, on ne peut ignorer d'autres influences : celles des pédagogies actives (on peut par exemple citer Dewey [4], qui pose l'Inquiry comme processus nécessaire à la construction du savoir en sciences), et celles des résultats de la recherche en didactique des sciences. De ces recherches ont émergé des propositions allant dans le sens d'un enseignement des sciences moins dogmatique et plus ouvert. L'enthousiasme des promoteurs de la DI, celui des maitres d'ouvrage (des enseignants, des formateurs) ne doit pas pour autant faire oublier que la DI est un objet questionné par la recherche, en éducation, en didactique et, de ce point de vue, les questions qu'elle suscite sont nombreuses. Qu'en est-il par exemple des références épistémologiques : la DI présente-t-elle des traits communs avec les démarches de la science, celle des laboratoires ? Est-ce important que cela soit, d'ailleurs ? Quels types de savoirs se prêtent au jeu d'une mise en scène sous forme de DI ? Quels sont les apprentissages effectivement réalisés ? Et qu'en est-il de la formation des enseignants? Quels outils peut-on leur fournir pour une mise en œuvre appropriée ? Et, finalement, quels seront les effets à long terme ? Autant de questions dont les réponses sont aujourd'hui loin d'être consensuelles.

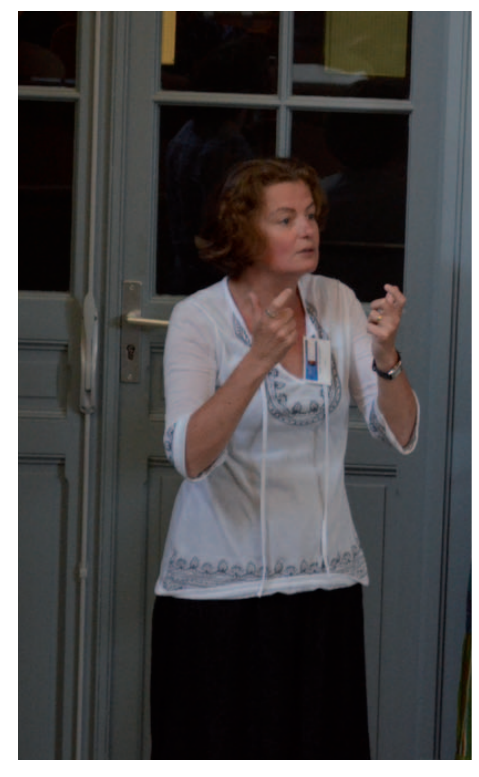

De gauche à droite : C. de Hosson, V. Munier, A. Delserieys et E. Blanquet.

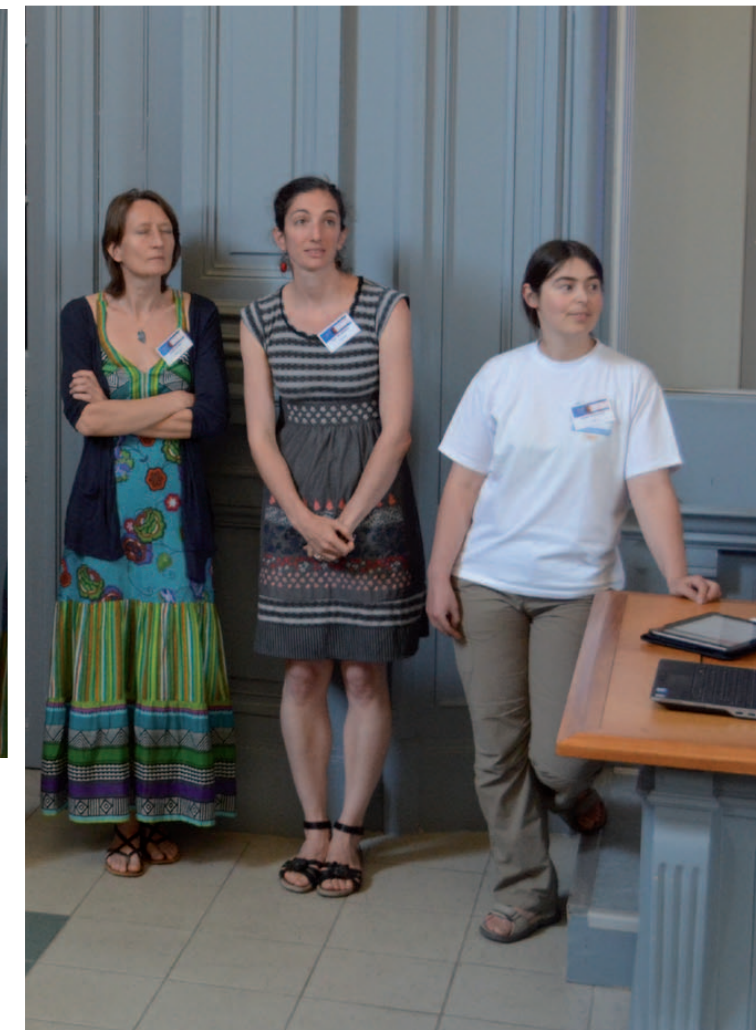

\section{La démarche d'investigation (DI) :}

\section{vers une tentative de définition...}

L'existence d'un label choisi pour désigner une approche pédagogique pourrait laisser penser qu'il existe une définition consensuelle et bien calibrée de ce que cette approche recouvre. On pourrait également s'attendre à ce qu'au label soit associé un processus standardisé, identifié et identifiable, ce qui est loin d'être le cas, la DI semblant, au contraire, échapper à toute tentative de modélisation. La diversité des formes prises par la DI semble faire écho à cette impossible gageure de définition. En effet, lorsqu'elles sont analysées par les chercheur.e.s en éducation, les séances de classe se réclamant de l'investigation présentent une variabilité notable. Si donc chacun (chercheurs, enseignants, formateurs) s'accorde aujourd'hui à considérer (et, d'une certaine façon, à assumer) la DI comme une approche pédagogique à facettes multiples, il n'en demeure pas moins que l'esprit général de cette approche apparaît gouverné par quelques mots, idées, principes relativement stables dans l'espace et dans le temps.

Ainsi, la DI promeut-elle un apprentissage plutôt coopératif, reposant sur l'initiative et le questionnement de l'élève. Ici, le savoir scientifique n'est pas transmis de manière verticale et descendante depuis le maître vers la classe, mais construit par les élèves tout au long d'un processus au sein duquel le " choix ", "l'identification » et la " résolution " d'un problème scientifique forment le cœur de l'activité (attendue) des élèves ; dans ce contexte, I'enseignant n'est plus exclusivement transmetteur du savoir, mais également médiateur. 


\section{\>>}

\section{Développer des compétences scientifiques à l'école maternelle $[5,6]$}

Les contenus enseignés à l'école maternelle sont très éloignés de ceux de la science professionnelle. Distinguer les activités susceptibles de relever de l'enseignement scientifique se révèle donc souvent difficile pour les enseignants. Peut-on développer chez les enfants d'école maternelle des compétences méthodologiques scientifiques et aider leurs enseignants à identifier comme scientifiques certaines pratiques de classe ?

Il a été proposé à environ 180 élèves de grande section de maternelle (5-6 ans) des activités en DI soigneusement conçues pour construire :

- la primauté de l'expérience au regard de l'opinion et de l'autorité,

- la reproductibilité,

- la robustesse (i.e. s'assurer qu'un changement mineur des conditions expérimentales ne change pas de façon spectaculaire le résultat),

- le recul (i.e. la distinction entre le monde physique et ses représentations),

- la formulation de généralisations.

Des interviews systématiques de binômes d'élèves réalisées après le travail en classe ont permis d'établir que l'idée de primauté de l'expérience est intégrée par une grande majorité des élèves. C'est aussi le cas, dans une certaine mesure, de celles de robustesse et de recul. Un résultat inattendu est la difficulté des élèves à intégrer la notion de reproductibilité en comparaison à celle de robustesse. L'acquisition de compétences plus sophistiquées semble en revanche dépendre de l'activité considérée et de l'expérience de l'enseignant.

Ces compétences méthodologiques peuvent être reformulées en critères de scientificité. Il est alors possible, en se fondant sur une approche bottom-up de la science scolaire, de construire des définitions de la science pour lesquelles le travail des élèves relève en toute légitimité de la pratique scientifique.

Les enseignants accueillent par ailleurs favorablement l'outil épistémologique élémentaire que constitue ce jeu de critères de scientificité. Des questionnaires ont établi qu'une majorité d'entre eux considère utile une liste explicite de compétences méthodologiques accessibles à leurs élèves. Beaucoup envisagent son utilisation comme un guide pour leur pratique de classe.
Les DI en mathématiques et en physique : place et rôle de la mesure dans les deux disciplines

Dans les programmes du secondaire, la DI est préconisée dans toutes les disciplines scientifiques, et les instructions officielles laissent supposer des démarches très voisines en physique et en mathématiques : les programmes accordent une place centrale à la résolution de problèmes, et on retrouve des finalités communes pour les DI dans les deux disciplines. Il s'agit non seulement de construire une culture scientifique commune et de contribuer à la formation des futurs citoyens, mais aussi de viser des enjeux d'ordre épistémologique.

Cependant, s'il existe des proximités des démarches, on ne peut pas dire pour autant qu'elles soient identiques : les modes d'élaboration des connaissances, et en particulier le rôle de la mesure dans ce processus, different dans les deux disciplines, voire au sein même d'une discipline. Par exemple, dans le cadre de la géométrie "pratique " (de l'école ou des professionnels) la validation se fait par la mesure, en prenant en compte les questions d'approximation. En physique la mesure occupe une place centrale dans les DI, notamment lorsque les élèves élaborent une loi à partir de données empiriques, ce qui nécessite aussi de prendre en compte les incertitudes liées au mesurage. En revanche, dans le cadre de la géométrie "théorique " l'activité de mesurage n'a pas de sens et l'élaboration des connaissances se fait via la démonstration. Les élèves sont ainsi confrontés à des situations dans lesquelles la mesure n'a pas le même statut, et on peut penser que cela peut être une source de difficultés.

Or, une réflexion sur le rôle de la mesure peut faire évoluer la vision de la science des élèves et les aider à mieux comprendre la nature de l'activité scientifique, ce qui est un des objectifs affichés de l'introduction des DI dans l'enseignement. On peut penser que les concepteurs de programmes en sont conscients, puisqu'ils accordent à la mesure et aux incertitudes une place croissante. Cependant, il existe un décalage entre les pratiques des enseignants et les prescriptions sur ces questions, notamment parce que certains enseignants ne maitrisent pas suffisamment les concepts en jeu. Cela explique en partie les difficultés des élèves et étudiants, pointées par plusieurs recherches, qui montrent que ceux-ci disposent de très peu des outils conceptuels permettant de raisonner sur les résultats de mesure.

Cela nous amène à préconiser l'introduction d'éléments d'épistémologie de la mesure dans les cursus scolaires, ce qui nécessite de développer la formation disciplinaire, didactique et épistémologique des enseignants sur ces questions.

\section{Enseignement universitaire et démarches d'investigation}

Avec l'évolution du baccalauréat en sciences physiques, l'enseignement en licence a été bousculé. Les étudiants n'ont pas le même rapport au formalisme mathématique, et seraient davantage formés à tenir un discours cohérent et argumenté dans une démarche expérimentale. Dans une logique ascendante, un enseignement des sciences par investigation a d'abord été préconisé à l'école primaire, au collège, puis au lycée. Celui-ci prend des formes différentes selon l'âge des élèves et la complexité des connaissances abordées, mais repose sur des principes communs : établir un lien avec des pratiques de scientifiques et passer d'une culture de transmission du savoir centrée sur l'enseignant à une culture d'appropriation centrée sur l'étudiant. Pourtant, que ce soit en France ou à l'étranger, peu de recherches font état d'expériences d'enseignement par investigation à l'université. Ces expériences sont souvent réservées à la formation des enseignants, ou témoignent d'investigations très guidées et limitées à une partie restreinte des enseignements. À l'inverse, une autre logique, descendante, vise à faire acquérir aux étudiants des compétences recherchées dans le monde professionnel : travailler avec les autres, planifier, organiser, gérer son propre travail. Depuis plus de 30 ans, cela se traduit par l'introduction d'approches par projet ou par problème dans certaines formations universitaires, en particulier les ingénieurs ou les professionnels de la santé.

L'enseignement de la physique à l'université, et particulièrement en licence, est tiraillé par ces logiques et la réponse n'est pas évidente. L'organisation traditionnelle - selon le découpage cours magistral, travaux dirigés, travaux pratiques - est régulièrement remise en cause, en particulier pour les difficultés récurrentes des étudiants à s'approprier des concepts de physique. Pourtant, d'après Henderson et Dancy [7], les freins à une évolution de l'enseignement 
universitaire ne se trouvent pas dans ce que pensent les enseignants d'autres modèles pédagogiques, mais dans l'organisation même des enseignements (programmes surchargés, manque de temps, salles inadaptées...). Par ailleurs, les enseignants à l'université sont généralement très impliqués dans leurs travaux de recherche, et ce double regard relève donc d'une acrobatie périlleuse pour le chercheur ou l'enseignantchercheur qui jongle avec plusieurs casquettes. De nombreux auteurs ont tenté de résoudre ce problème en invoquant un "research teaching nexus" [8]. Ainsi, un équilibre serait trouvé dans un enseignement fondé sur la recherche (research-based teaching), c'est-à-dire qui aborde, avec les étudiants, des questions nouvelles pour la discipline elle-même.

(a) Pour mémoire, ce dispositif d'accompagnement pour l'enseignement des sciences, créé en 1996 par

G. Charpak, avait pour objectif de mobiliser les différents acteurs de l'éducation sur la question de l'insuffisance de l'École en terme d'enseignements scientifiques. Les racines de La main à la pâte sont à rechercher de l'autre côté de l'Atlantique, à Chicago, où s'installait le programme Hand's On qui devait remobiliser les élèves des quartiers défavorisés [9].

\section{Références}

1• A. Pedregosa, « Dans le sillage de la main à la pâte. Rencontre entre disciplines scientifiques au collège ", Reflets de la Physique, 14 (2009) 20-22

2•S. Mathé, M. Méheut et C. de Hosson, « Démarche d'investigation au collège : quels enjeux ?", Didaskalia, 32 (2008) 41-76.

3• M. Rocard et al., « L'enseignement scientifique aujourd'hui : une pédagogie renouvelée pour l'avenir de l'Europe » (2007) (http://ec.europa. eu/research/sciencesociety/document_library/ pdf_06/report-rocard-on-science-education_fr.pdf).

4• J. Dewey, Logic: The Theory of Inquiry, The later works, Vol. 12 (1938), Southern Illinois University (USA).

5• E. Blanquet, Sciences à lécole côté jardin, le quide pratique de l'enseignant, Éditions du Somnium, Nice (2010).

6• E. Blanquet, "Faire et refaire, ou l'art de varier les paramètres avec les tout-petits", dans Réveille-moi les sciences!, Éditions De Boeck, Belgique (2012).

7• C. Henderson et M.H. Dancy, "Barriers to the use of research-based instructional strategies: The influence of both individual and situational characteristics", Phys. Rev. Special Topics - Physics Education Research, 3 (2007) 020102

$8 \bullet$ A. Brew et D. Boud, “Teaching and research: establishing the vital link with learning", Higher Education, 29 (1995) 261-273.

9• G. Charpak, La main à la pâte. Histoire des sciences à l'école primaire, Flammarion, Paris (1998). 